\title{
Article \\ A Novel Deep Learning Model to Predict Ultimate Strength of Ship Plates under Compression
}

\author{
So-jeong Cho $\mathbb{D}$, Im-jun Ban $(\mathbb{B}$ and Sung-chul Shin *(i) \\ Department of Naval Architecture \& Ocean Engineering, Pusan National University, Busan 46241, Korea; \\ csj2612@pusan.ac.kr (S.-j.C.); june3373@pusan.ac.kr (I.-j.B.) \\ * Correspondence: scshin@pusan.ac.kr; Tel.: +82-51-510-2525
}

Citation: Cho, S.-j.; Ban, I.-j.; Shin, S.-c. A Novel Deep Learning Model to Predict Ultimate Strength of Ship Plates under Compression. Appl. Sci. 2022, 12, 2522. https://doi.org/ $10.3390 /$ app 12052522

Academic Editor: José A. Orosa

Received: 15 December 2021

Accepted: 25 February 2022

Published: 28 February 2022

Publisher's Note: MDPI stays neutral with regard to jurisdictional claims in published maps and institutional affiliations.

Copyright: (C) 2022 by the authors. Licensee MDPI, Basel, Switzerland. This article is an open access article distributed under the terms and conditions of the Creative Commons Attribution (CC BY) license (https:// creativecommons.org/licenses/by/ $4.0 /)$.

\begin{abstract}
The evaluation of ship strength during the hull structure design stage is essential for structural safety. Additionally, plate is one of the basic structural members of the hull, it is important to analyze and predict the ultimate strength. The curved plate used for shipbuilding must be able to withstand repeated axial loads and complex loads, and stability needs to be confirmed through ultimate strength analysis. In general situations, the magnitude of the transverse compression is smaller than that of the longitudinal and combined loads, but transverse compression causes different physical behaviors from the longitudinal load state, which affects the ultimate strength, so study on the ultimate strength of the curved-plate under transverse compression is essential. Therefore, in this paper, a curved plate under transverse compressive load was selected as a subject, and the ultimate strength of the curved plate under the corresponding compression condition was predicted using a deep learning model. To obtain the training data for the deep-learning model, 4050 cases were selected and analyzed using the ANSYS. The accuracy of the model was verified by comparing the results predicted by the model and empirical equations with those of the FEM analysis. The study shows that it is possible to consider the ultimate strength more efficiently in the initial design stage of the ship.
\end{abstract}

Keywords: ultimate strength; curved plate; deep neural network

\section{Introduction}

Due to the increasing number of ships constructed every year, the international maritime traffic has grown rapidly. Curved plates are one of the basic structural elements of hulls. However, in recent years, owing to the reduction in the weight of ships, extremely thin curved plates have become vulnerable to buckling and corrosion [1]. Therefore, it is important to analyze buckling and collapse of structural elements such as curved plates.

The curved plates used in ship construction must be able to withstand repeated axial and complex loads; hence, its stability is confirmed by estimating the ultimate strength. The transverse compressive force is generated by the difference between the hydrostatic pressure of the ship and the pressure due to the weight of the cargo.

Generally, the magnitude of the transverse compressive force is smaller than that of the longitudinal compressive force [2]. However, during the transverse and combined loading states, a physical behavior occurs which is different from that of the longitudinal loading. This acts as a factor influencing the ultimate strength, which is an essential part of this research. Therefore, in this study, curved plates subjected to transverse compressive loads were used as the research subjects.

There are three strategies through which the ultimate strength can be estimated: a finite element method (FEM) universal analysis program, an experimental approach, and an empirical formula derived from the experimental results [3]. However, a new analysis must be conducted when the dimensions or materials of the plate are changed during the design stage. For instance, in the case of the empirical formula method, the ultimate 
strength of the curved plate differs from the actual analysis result if the usable range is limited or if the curved plate undergoes nonlinear behavior, resulting in low reliability.

It is an advantage of a deep learning model to be able to find regularity through statistical analysis of parameters and result values within the data. Using this, the ultimate strength considering nonlinear behavior is estimated, and the appropriateness and necessity of the deep learning model are suggested compared with the empirical equations presented from previous studies [4].

In a previous study, $\mathrm{Pu}$ and Mesbahi [5] developed a model for predicting the ultimate strength of a plate with a single-hidden-layer perceptron neural network. Upon comparing the model with the existing empirical formula, the predicted result using the neural network yielded a value closer to the actual experimental value. This result confirms that the deep learning-based model can be useful during the initial design stage; however, the neural network used was a single-layer perceptron neural network, and a relatively small number of cases were used with 143 training datasets. In this study, based on a deep neural network, the ultimate strength of the curved plate was predicted over a wider range under a transverse load in various scenarios. These results indicate the ability of the deep neural network to efficiently estimate the ultimate strength to acquire the optimal design of the ship and cost reduction in the initial design stage.

Several studies have been conducted to predict the ultimate strength of structural members using ANN. Zareei et al. [6] estimated the ultimate strength of stiffened aluminum plates with flat-bar stiffeners under in-plane longitudinal compression loads using an ANN. When comparing this value with the ultimate strength obtained using empirical equations, it was confirmed that the model prediction was close to the FEM result. However, the number of cases in this study was limited (42 datasets). Thus, these results cannot be considered sufficiently robust, since prediction models require a large variety of datasets to perform effectively over a wide range of applications.

Khalaf et al. [7] developed an ANN model to predict the ultimate strength of circular concrete-filled steel tube columns under concentric loading. A model comprising two hidden layers and 20 nodes was trained for 10,000 epochs. Out of a total of 280 datasets, 224 datasets, that is, $80 \%$, were used as training data, while the remaining $20 \%$ were used as test data. The suitability and accuracy of the model were verified by comparing the coefficient of determination obtained from the empirical equations and that from the predictions. The comparison showed that ANNs can effectively predict the physical properties of structural components.

Previous studies on ANNs to predict the physical properties of structural members have shown reliable results in terms of speed and accuracy. However, existing studies are limited, in that there are few scenarios of structural members used for learning, which cannot be applied to multiple cases. For a wide range of applications, it is necessary to obtain data from different scenarios under various conditions and derive results through learning.

\section{Methodology}

\subsection{Research Method}

Prior to constructing a neural network model to generate data for use in deep neural network learning, a case used for hull construction in a shipyard and a curved plate showing nonlinear behavior were selected and studied using ANSYS, which is a universal analysis program.

In this study, 4050 cases were selected and analyzed by defining the factors affecting the ultimate strength of the curved plate. The load was set to a transverse compressive load condition. The boundary conditions, as shown in Figure 1, were applied as simply supported conditions on the four sides, and the plate was maintained to be flat with no curvature. The magnitude of the pressure applied to the plate is yield stress*thickness ${ }^{*} 2$. The verification of the analytical model and the boundary conditions was confirmed by the results of a previous study. 


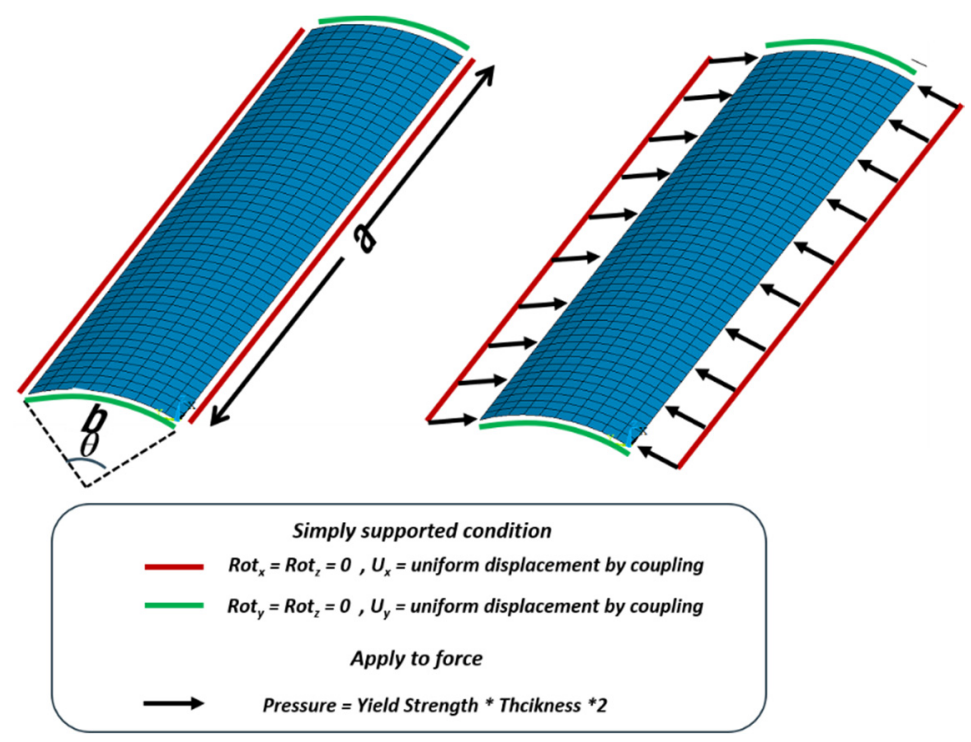

Figure 1. Applied boundary condition in curved plates.

The suitability of deep neural network learning was examined through a correlation coefficient analysis using geometric figures, shape ratios, and material properties of the curved plate as parameters. A neural network model was then constructed using the selected parameters and results, and the obtained data were combined with training and test data to compare the errors with the finite element analysis results. This paper is based on the conclusions derived from these results.

\subsection{Curved Plate Analytical Modeling Scenarios and Analysis Techniques}

The analytical model scenario to be used as the training data for the neural network was selected considering the three initial deflection amounts proposed by Smith et al. [8] in Equation (1). Where $w_{0 p l}$ refer to maximum values of representative initial deflections, $\beta$ refers to plate slenderness ratio, and $t$ refers to plate thickness.

A total of 4050 scenarios were selected by combining geometric shapes, length, curvature, and thickness of the curved plate. The thickness of the plate was selected according to the yield strength of the material considering the curvature $\left(1-9^{\circ}\right)$ and plate slenderness range (1-5) in the curved plate.

$$
w_{0 p l}= \begin{cases}0.025 \beta^{2} t & \text { for slight level } \\ 0.1 \beta^{2} t & \text { for average level } \\ 0.3 \beta^{2} t & \text { for severe level }\end{cases}
$$

The material properties and scenario of curved plate used in the analysis are listed in Tables 1 and 2, respectively. The arc-length method was applied to observe the nonlinear behavior, such as complex buckling deformation.

Table 1. Material properties for curved-plate.

\begin{tabular}{cc}
\hline Elastic Modulus & Poisson's Ratio \\
\hline $205.8 \mathrm{GPa}$ & 0.3 \\
\hline
\end{tabular}


Table 2. Scenario of curved-plate.

\begin{tabular}{cc}
\hline Yield Stress (MPa) & Thickness (mm) \\
\hline 235 & $7,10,13.5,16.5,20,24,28.5,32,36.5,42$ \\
315 & $8.5,12,15.5,18.5,22,26,30,34,38.5,44.5$ \\
355 & $9,12,17,19,23,26.5,30.5,34.5,42,50$ \\
\hline
\end{tabular}

A modal analysis was performed using ANSYS because the curved plate exhibited a shape different from the initial deflection shape of the flat plate. Figure 2 shows the shape of the 1st-mode analysis, with the initial deflection of the curved plate applied. In the curved plate, $b=1000 \mathrm{~mm}$, aspect ratio $=3$, material yield stress $=235 \mathrm{MPa}$, curvature $=9^{\circ}$, thickness $=13.5 \mathrm{~mm}$ and under transverse compression.

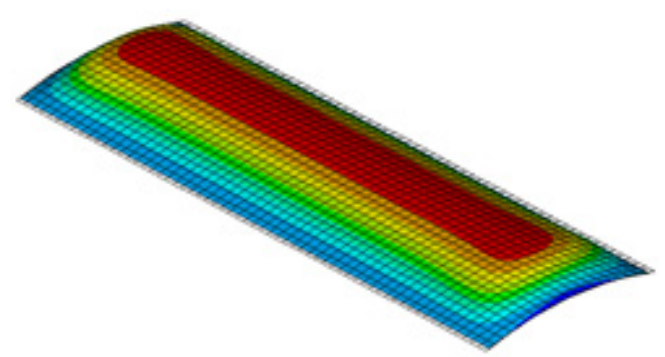

Figure 2. Buckling under uniaxial compression.

\subsection{Grid Convergence Study and Initial Deflection Application}

In the FEM analysis, the size of the grid significantly affects the analysis results [9]. Additionally, because initial deflections due to welding and thermal processing are detrimental to plate structures, initial deflection must be provided for accurate analysis results [10]. A modal analysis was performed and applied as the curved plate had a shape different from the initial deflection shape of the plate due to the influence of the curvature. The analysis was applied in the first mode shape in the analysis results because it was the most deformable with minimum strain energy. Figure 3 shows the shape of the initial deflection according to the curvature.

\begin{tabular}{|c|c|c|c|c|c|}
\hline \multicolumn{6}{|c|}{ Initial deflection of curved plate } \\
\hline & & & & & $\left(\theta=\begin{array}{rl}(\text { Flank angle }) \\
\text { Scale }=200\end{array}\right.$ \\
\hline$(\theta=1)$ & $\Longrightarrow$ & $(\theta=4)$ & $\Longrightarrow$ & $(\theta=7)$ & $\Longleftarrow$ \\
\hline$(\theta=2)$ & $\Longrightarrow$ & $(\theta=5)$ & $\Longrightarrow$ & $(\theta=8)$ & $\Longleftarrow$ \\
\hline$(\theta=3)$ & $\Longrightarrow$ & $(\theta=6)$ & $\rightleftharpoons$ & $(\theta=9)$ & $\Longleftarrow$ \\
\hline
\end{tabular}

Figure 3. Initial deflection of curved plate.

In addition to the effect of the initial deflection of the curved plate, the size of the grid also affects the FEM result. The denser the grid size, the closer the FEM value is to the results using the actual experiment. The result from the grid convergence shows that the denser the lattice, the lower is the stress. If the number of grids is insufficient, the result overestimates the ultimate strength of the plate, leading to inaccurate analysis results. In contrast, if the number of grids is excessively large, each node point generated by the grid increases dramatically, thereby increasing the analysis time and decreasing the efficiency [11]. The model of this study was created by fixing 20 square grids in the width direction, similar to the grid convergence survey of a previous study by Ban [12]. Figure 4 
shows the results of the ultimate strength analysis according to the number of elements along the width of the curved plate. According to the analysis model used in this study, the curved plate with 20 elements was selected for analysis. The details of the plates used in the analysis are provided in Figure 4 . NoE is the number of elements along the width. For different curved plates subjected to identical conditions, these values were set to $6,8,10,12$, 14,20 , and 30 to investigate the results of each ultimate strength analysis. Figure 4 shows that the value converges at $20 \mathrm{NoE}$. Thus, the required grids were created using this value.

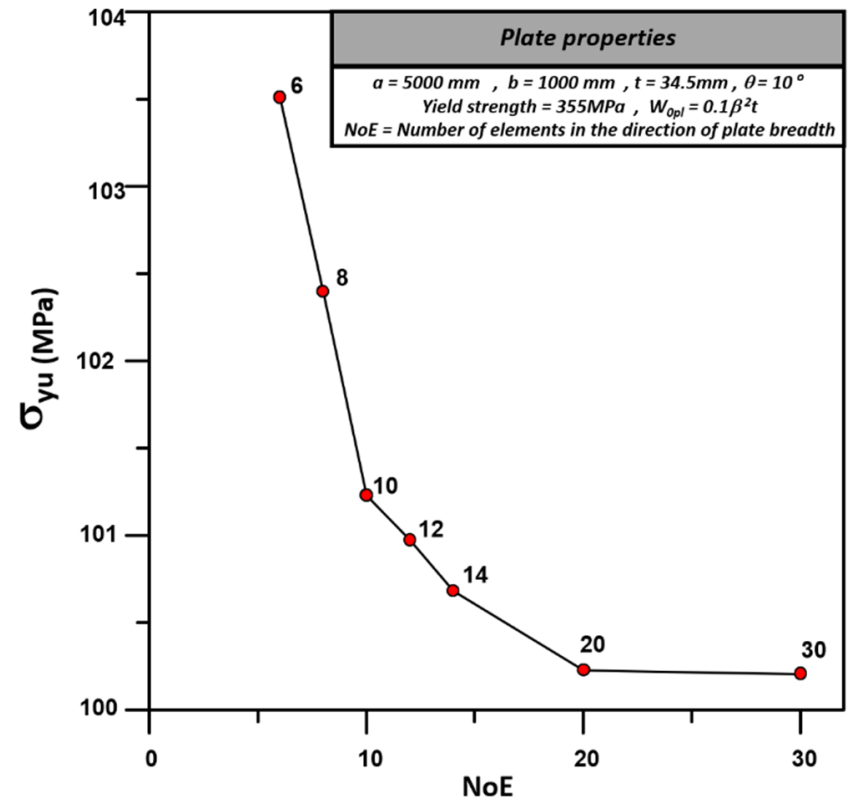

Figure 4. Mesh convergence study result.

\subsection{Deep Learning Model Configuration}

Deep learning differs from machine learning in that it can independently learn data. It is an artificial neural network composed of an input layer, a hidden layer, and an output layer, and can be used to model nonlinear relationships [13].

For the deep neural network model to predict the ultimate strength of a plate, a total of six parameters were selected through correlation analysis, and a Tensorflow-based Python program was used. The selected parameters are the initial deflection amount, plate slenderness ratio, plate length, plate thickness, curvature, and yield stress.

In this study, the loss function used in the deep learning model had the smallest mean square error, and the degree of learning was evaluated using this value. The loss function quantifies the difference between the actual and predicted values [14]. Given that the activation function can solve the vanishing gradient problem of the sigmoid function and has a fast convergence speed, the ReLU function used in most neural networks was applied [15]. Although various types of optimization functions are being used, optimization was performed using the most commonly used, the adaptive moment estimation (Adam) [16].

As presented in Table 3, the model was configured such that the weights from six input features and seven hidden layers received values from the activation function, ReLU, and output the ultimate strength value. Among a total of 4050 cases of data, $80 \%$ or 3240 cases were used as training data, and the training was conducted for 1500 epochs. The training data were used in the process of learning the model, and the test data evaluated the final performance of the model and whether the training was successful [17]. An epoch is the number of times the entire training set passes through the neural network. Specifically, one epoch indicates that the entire training set is applied to one neural network and passes through the neural network once through forward propagation and then through 
backward propagation [18]. The results were confirmed using 810 cases as the test data, which excluded the training data from the entire dataset.

Table 3. Deep learning model structure.

\begin{tabular}{cc}
\hline \multicolumn{2}{c}{ DNN Model Structure } \\
\hline Number of hidden layers \\
Number of nodes & 7 \\
Activation function & 156 \\
Input features & ReLU \\
Epoch & 6 \\
No. Training set & 1500 \\
No. test set & 3240 \\
\hline
\end{tabular}

\section{Analysis Results}

According to the analysis results of 4050 cases, it was confirmed that as the plate slenderness ratio increased, the ultimate strength decreased due to a decrease in thickness.

As shown in Figure 5, the ultimate strength changes according to the plate slenderness ratio material with a yield stress of $355 \mathrm{MPa}, \mathrm{b}=1000 \mathrm{~mm}$, an aspect ratio of three, and a curvature of $4^{\circ}$ under a transverse compressive load. The average value of initial deflection was applied.

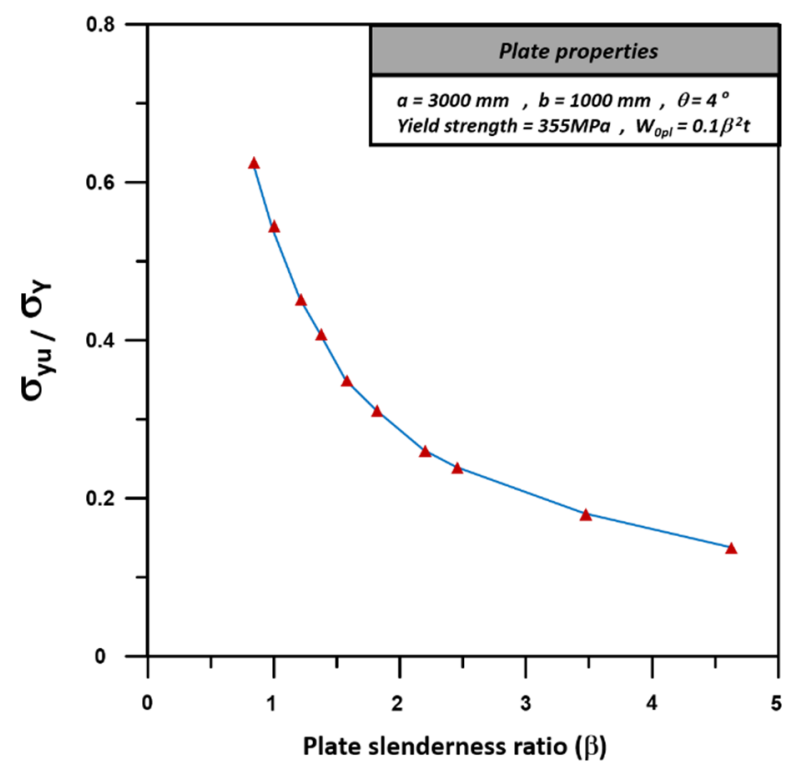

Figure 5. Effect of slenderness ratio on the analysis result.

As shown in Figure 6, the result of the ultimate strength changes according to Equation (1), which is the maximum value of the initial deflection. The three methods were applied according to the curved plate analysis scenario. Figure 6 also shows a graph of the S-S curve according to the degree of the initial deflection of a curved plate with $b=1000 \mathrm{~mm}$, an aspect ratio of 4 , a material yield stress of $315 \mathrm{MPa}$, and a curvature of $5^{\circ}$. As shown in Figure 6, when a slight level value with the smallest initial deflection value is applied, the highest ultimate strength is exhibited compared to the slenderness of the same plate, depending on how small the initial deflection value was, and the tendency to decrease is in the order of average and severe levels. 


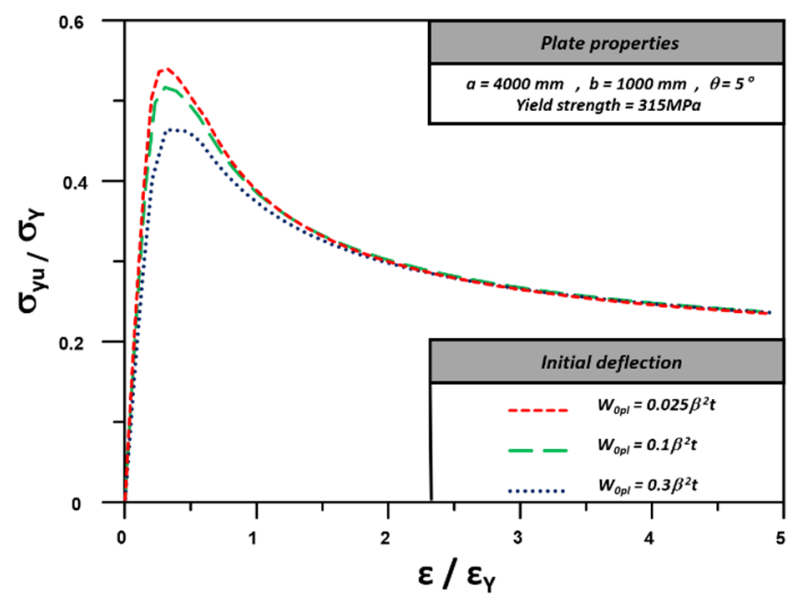

Figure 6. Effect of initial deflection ratio on the analysis result.

As shown in Figure 7, the ultimate strength changes according to the curvature of a curved plate with a yield stress of $235 \mathrm{MPa}$, an aspect ratio of four, and a thickness of 20 $\mathrm{mm}$. The average value of initial deflection was applied, however; unlike the curved plate subjected to a longitudinal compressive load, the graph shows that the ultimate strength decreases as the curvature increases.

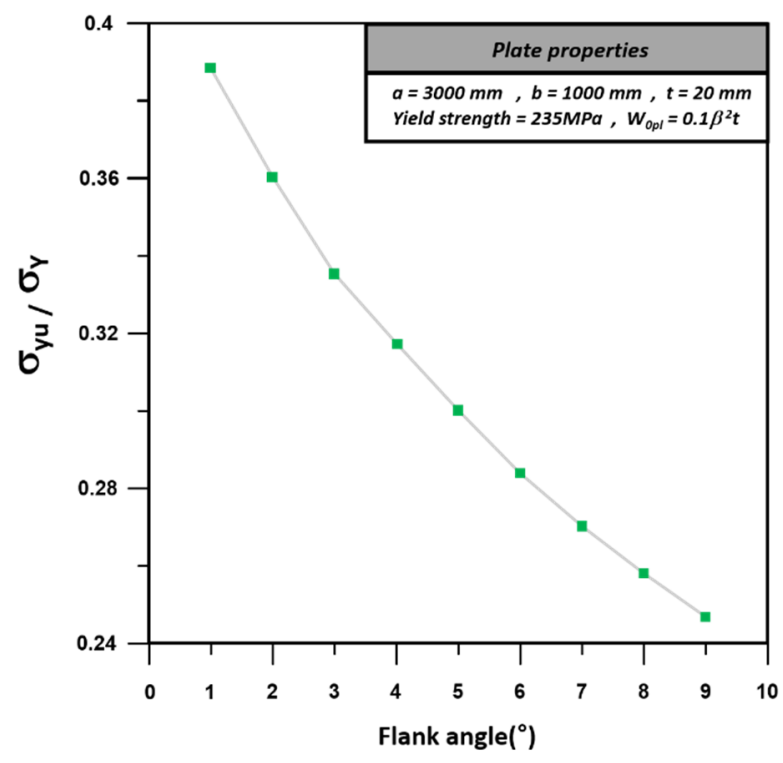

Figure 7. Effect of flank angle on the analysis result.

As shown in Figure 8, the ultimate strength of the curved plate gradually decreases as the aspect ratio increased. A curved plate with a width of $1000 \mathrm{~mm}$, thickness of $30 \mathrm{~mm}$, curvature of $9^{\circ}$, and yield stress of $315 \mathrm{MPa}$ was used, and an average value was applied for the degree of the initial deflection. 


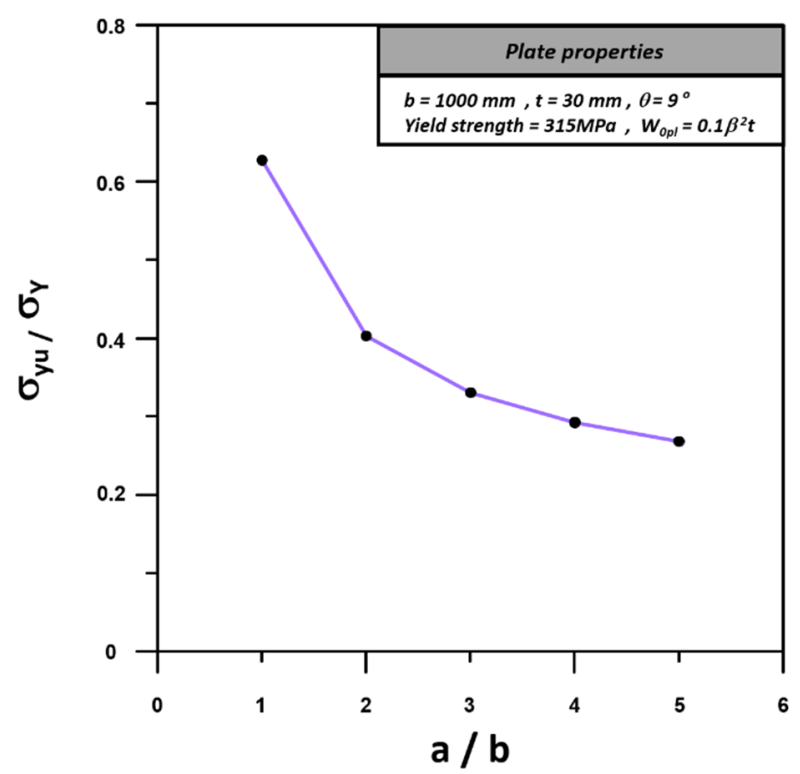

Figure 8. Effect of aspect ratio on the analysis result.

\section{Prediction Result Using Deep Learning Model}

\subsection{Prediction Result of Curved Plate Ultimate Strength}

The prediction results were examined using 810 test data cases for the model trained using deep neural network learning. Figure 9 shows the error rate for learning according to the epoch value, which is the number of learning times for all data.

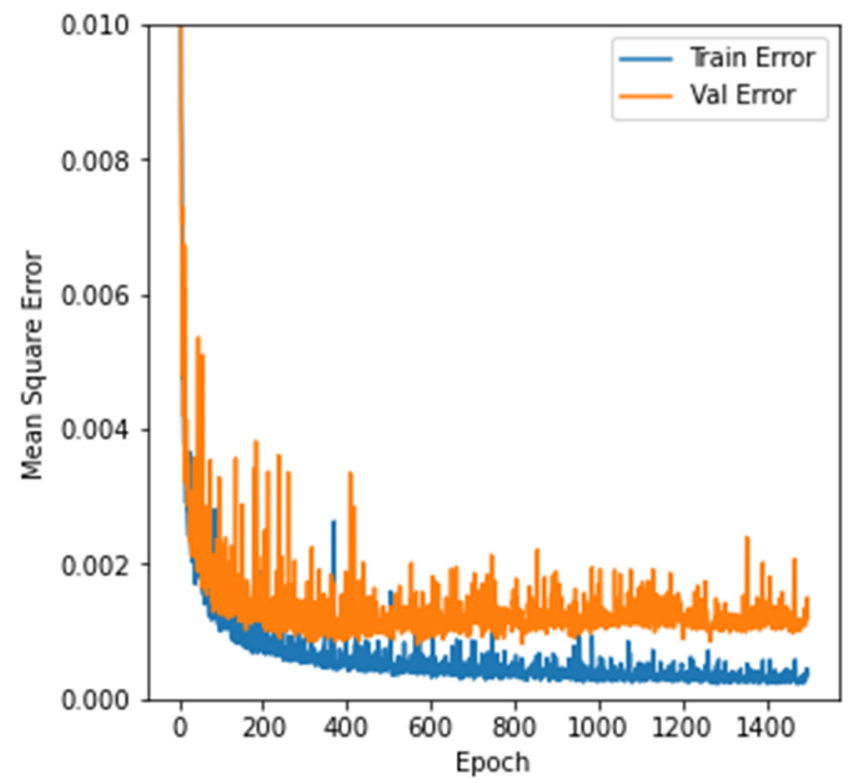

Figure 9. Deep learning model training result.

Figure 10 shows the coefficient of determination of the deep-learning model used in this study. Most of the predicted values are located close to the straight line representing the analysis results. The coefficient of determination is one of the indicators of the goodness of fit of the regression model. It can be defined as the square of the correlation coefficient and can be considered an indicator of the causality between the predictor $x$ and dependent variable $y$. 


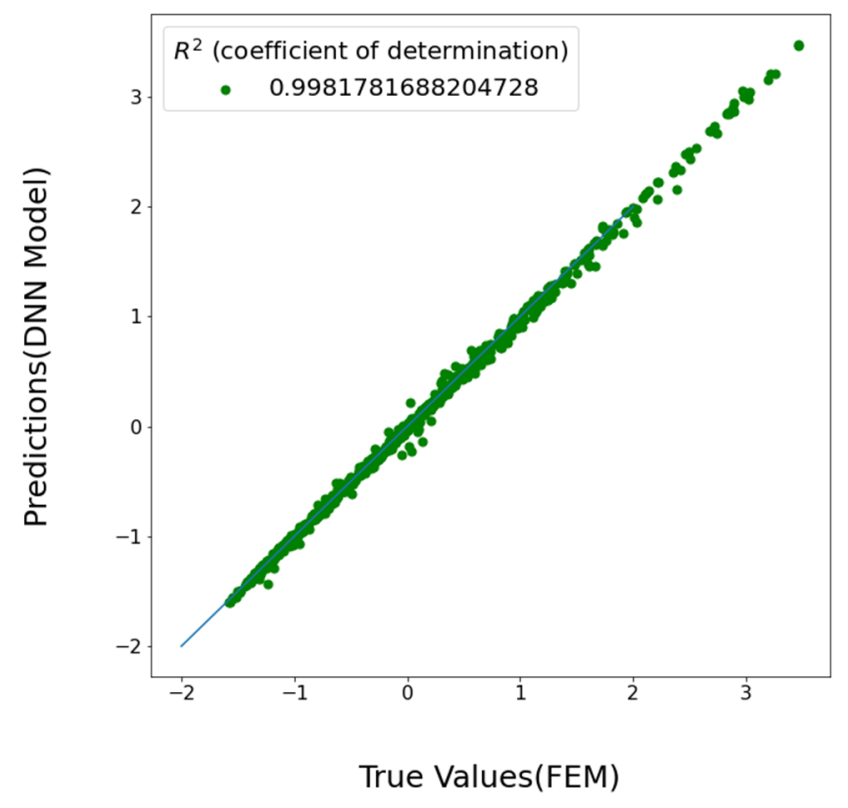

Figure 10. Coefficient of determination of deep learning model (all test data).

The values of the coefficient of determination range from 0 to 1 . The closer the value is to 0 , the lower the fit of the regression model, and the closer it is to 1 , the higher the fit of the model, that is, the better the model explains the data. The coefficient of determination of the deep-learning model conducted in this study is approximately 0.998 , which is close to 1 . Thus, it can be concluded that the model fits well.

\subsection{Comparison with the Empirical Formulae}

Equation (2) is an empirical formula proposed by Kim et al. [19] in previous studies that can predict the ultimate strength of a curved plate under transverse compressive load. In Equation (2), $\sigma_{U_{-} T C}$ is ultimate strength of the curved plate under transvers compressive load and $\sigma_{Y}$ is yield stress of material. In Equation (3), coefficient $C_{F}$ was assumed as a function of the double slenderness ratio, and the correction factors in Equation (4) represented the effect of curvature with the change in the thickness of the plate. $R$ in Equation (4) is the radius of curvature.

$$
\begin{gathered}
\frac{\sigma_{U_{-} T C}}{\sigma_{Y}}=\left(\frac{2.25}{\beta}-\frac{1.25}{\beta^{2}}\right) \times C_{F} \\
C_{F}=\frac{C_{a}}{\beta^{2}}+\frac{C_{b}}{\beta}+C_{c} \\
C_{a}=2.596\left(\frac{R}{t}\right)^{2}-1.712\left(\frac{R}{t}\right)+0.415 \\
C_{b}=-2.095\left(\frac{R}{t}\right)^{2}+0.929\left(\frac{R}{t}\right)-0.136 \\
C_{c}=1.009\left(\frac{R}{t}\right)^{2}-0.724\left(\frac{R}{t}\right)+0.322 \\
\sigma_{E}=K_{t g} \frac{\pi^{2} E}{12\left(1-v^{2}\right)}\left(\frac{t}{a}\right)^{2}
\end{gathered}
$$

where

$$
K_{t g}= \begin{cases}\frac{a}{\sqrt{R t}}+3 \frac{(R t)^{0.175}}{a^{0.35}} & \text { if } \frac{a}{R} \leq 1.63 \sqrt{\frac{R}{t}} \\ 0.3 \frac{a^{2}}{R^{2}}+2.25\left(\frac{R^{2}}{a t}\right)^{2} & \text { if } \frac{a}{R}>1.63 \sqrt{\frac{R}{t}}\end{cases}
$$


Equation (5) was proposed by DNVGL [20] as an empirical equation used to compare the results using the deep learning model in this paper. In the equation, $\sigma_{E}$ is the elastic buckling stress, and $a$ represents the length of the curved plate. The elastic modulus and Poisson ratio used in the equation were substituted with the values shown in Table 1, and the values obtained through Equation (6) were used for Buckling factor $K_{t g}$.

$$
\sigma_{c r}=\left\{\begin{array}{cl}
\sigma_{E} & \text { for } \sigma_{E} \leq 0.5 \sigma_{Y} \\
\sigma_{Y}\left[1-\frac{\sigma_{Y}}{4 \sigma_{E}}\right] & \text { for } \sigma_{E}>0.5 \sigma_{Y}
\end{array}\right.
$$

In many cases, elastic buckling stress needs to be corrected because the value calculated by the above method is higher than the yield stress. Therefore, critical buckling stress was calculated through the Johnson-Ostenfeld method of Equation (7), and proportional limit was assumed to be a commonly used $0.5 \sigma_{Y}$ [21].

In this study, the presented empirical equations and values predicted using the DNN model were compared. Comparisons were made between the results of this study and those of the studies by Kim et al. [19] and DNVGL [20]. Table 4 shows the error between these prediction results and FEM values. Because the geometric ranges of the curved plate applicable to each empirical equation is different, the conditions of the curved plates were selected and compared by referring to studies that proposed the empirical equation.

Table 4. Comparison of the deep learning model result and empirical formulae.

\begin{tabular}{cc}
\hline Empirical Formulae and DNN Model & Error (\%) \\
\hline Kim et al. (2014) & 12.3351 \\
DNVGL (2015) & 33.1862 \\
DNN & 1.2648 \\
\hline
\end{tabular}

The empirical formulae presented by Kim et al. [19] and DNVGL [20] showed average error rates of $12.33 \%$ and $33.19 \%$, respectively, and both showed a larger error rate as the aspect ratio, curvature, and initial deflection increased. In contrast, in the case of ultimate strength prediction using deep learning, the average error rate was approximately 1.26\%, indicating a higher accuracy than the empirical formulae.

\section{Conclusions}

In this study, a nonlinear analysis of a curved plate scenario subjected to a transverse compressive load was performed using ANSYS, and a deep learning model was developed to predict the ultimate strength using the obtained analysis results as learning data. The prediction of the ultimate strength for 810 cases (20\%) out of 4050 analysis scenarios, created according to the geometrical values and physical properties of the curved plate, showed a high accuracy of approximately $98.74 \%$. The following conclusions were derived based on the study results.

(1) It is possible to estimate the ultimate strength of a curved plate using deep learning models faster than when using the FEM.

(2) Deep learning models can be applied more widely than the method of estimating the ultimate strength using empirical formulae.

(3) Deep learning models exhibit high accuracy as the ultimate strength prediction result of the curved plate.

Although a deep learning model was developed using the analysis results of 4050 curved plates, it is expected to be more useful for estimating the ultimate strength in the actual design stage if the analysis results are applied under more diverse conditions for data learning, such as a stiffened curved plate or a curved plate under combined load conditions. 


\begin{abstract}
Author Contributions: Conceptualization, S.-j.C., I.-j.B. and S.-c.S.; Data curation, S.-j.C. and I.-j.B.; Formal analysis, S.-j.C., I.-j.B. and S.-c.S.; Investigation, S.-j.C. and I.-j.B.; Methodology, S.-j.C. and I.-j.B.; Writing—original draft, S.-j.C.; Writing—review and editing, S.-j.C. and I.-j.B.; Project administration, S.-c.S.; Supervision, S.-c.S. All authors have read and agreed to the published version of the manuscript.
\end{abstract}

Funding: This research received no external funding.

Institutional Review Board Statement: Not applicable.

Informed Consent Statement: Not applicable.

Data Availability Statement: Not applicable.

Acknowledgments: This research was supported by the Korea Institute for Advancement of Technology (KIAT) grant funded by the Korea Government (MOTIE) (No. P0001968, Competency Development Program for Industry Specialists).

Conflicts of Interest: The authors declare no conflict of interest.

\title{
References
}

1. Lee, J.W.; Kim, S.G.; Park, J.S.; Chun, M.S.; Suh, Y.S.; Lee, J.M. Development of advanced formulation to predict the buckling/ultimate strength of curved plates subjected to combined axial compression and lateral pressure. J. Soc. Nav. Archit. Korea 2011, 6, 1766-1769.

2. Kwen, Y.W. The Development of Buckling and Ultimate Strength Formulae for Ship Curved Plates. Master's Thesis, Pusan National University, Pusan, Korea, 2004.

3. Paik, J.K.; Amlashi, H.; Boon, B.; Branner, K.; Caridis, P.; Das, P.; Fujikubo, M.; Huang, C.-H.; Josefson, L.; Kaeding, P.; et al. Committee III.1 Ultimate Strength. In 18th International Ship and Offshore Structures Congress (ISSC 2012), Proceedings of the 18th International Ship and Offshore Structures Congress, Rostock, Germany, 10-13 September 2012; Fricke, W., Bronsart, R., Eds.; Schiffbautechnische Gesellschaft e.V.: Hamburg, Germany, 2012; pp. 285-363.

4. Sze, V.; Chen, Y.; Yang, T.; Emer, J.S. Efficient Processing of Deep Neural Networks: A Tutorial and Survey. Proc. IEEE 2017, 105, 2295-2329. [CrossRef]

5. Pu, Y.; Mesbahi, E. Application of artificial neural networks to evaluation of ultimate strength of steel panels. Eng. Struct. 2006, 28, 1190-1196. [CrossRef]

6. Zareel, M.R.; Khedmati, M.R.; Rigo, P. Application of artificial neural networks to the evaluation of the ultimate strength of uniaxially compressed welded stiffened aluminum plates. Proc. Inst. Mech. Eng. Part M J. Eng. Marit. Environ. 2012, 226, 197-213.

7. Khalaf, A.A.; Naser, K.Z.; Kamil, F.N. Predicting the Ultimate Strength of Circular Concrete Filled Steel Tubular Columns by Using Artificial Neural Networks. Int. J. Civ. Eng. Technol. 2018, 9, 1724-1736.

8. Smith, C.S.; Davidson, P.C.; Chapman, J.C. Strength and Stiffness of ships' Plating under In-Plane Compression and Tension. R. Inst. Nav. Archit. Trans. 1988, 130, 277-296.

9. Liu, Y.; Glass, G. Effects of Mesh Density on Finite Element Analysis; SAE International: Detroit, MI, USA, 2013.

10. Paik, J.K. Ultimate Limit State Analysis and Design of Plated Structures, 2nd ed.; Wiley: Chichester, UK, 2018 ; pp. 43-56.

11. More, S.T.; Bindu, R.S. Effect of mesh size on finite element analysis of plate structure. Int. J. Eng. Sci. Innov. Technol. 2015, 4, 181-185.

12. Ban, I.J. A study on the Effect of Number of Elements for Assessing Ultimate Strength. Master's Thesis, Pusan National University, Pusan, Korea, 2021.

13. Kim, H.S.; Ahn, I.G.; Kim, Y.I. Development of a fatigue damage model of wideband process using an artificial neural network. J. Soc. Nav. Archit. Korea 2015, 52, 88-95. [CrossRef]

14. Kang, M.J. Comparison of gradient descent for deep learning. J. Korea Acad. Ind. Coop. Soc. 2020, 21, 189-194.

15. Lee, D.C.; Park, B.J. Comparison of deep learning activation functions for performance improvement of a $2 \mathrm{D}$ shooting game learning agent. J. Inst. Internet Broadcast. Commun. 2019, 19, 135-141.

16. Kim, S.Y.; Chung, W.K.; Shin, S.R. Acoustic Full-waveform Inversion using Adam Optimizer. Geophys. Geophys. Explor. 2019, 22, 202-209.

17. Kraus, M.; Feuerriegel, S. Decision support from financial disclosures with deep neural networks and transfer learning. Decis. Support Syst. 2017, 104, 38-48. [CrossRef]

18. Buscombe, D.; Carini, R.J.; Harrison, S.R.; Chickadel, C.C.; Warrick, J.A. Optical wave gauging using deep neural networks. Coast. Eng. 2020, 155, 103593. [CrossRef]

19. Kim, J.H.; Lee, K.H.; Kim, J.H.; Kim, M.H.; Lee, J.M. Computational analysis and design formula development for the design of curved plates for ships and offshore structures. Struct. Eng. Mech. 2014, 49, 705-726. [CrossRef]

20. DNVGL. BUCKLING. 2015. Available online: https://rules.dnvgl.com/docs/pdf/DNVGL/CG/2015-10/DNVGL-CG-0128.pdf (accessed on 1 October 2015).

21. Park, J.S.; Seo, J.K. Modified Design Formula for Predicting the Ultimate Strength of High-tensile Steel Thin Plates. J. Korean Soc. Mar. Environ. Saf. 2021, 27, 447-456. [CrossRef] 